\title{
PHILIPPE CASSOU-NOGUÈS
}

\section{ANUPAM SRIVASTAV \\ On Taylor's conjecture for Kummer orders}

Séminaire de Théorie des Nombres de Bordeaux, tome 2, nº 2 (1990), p. $349-363$

<http://www.numdam.org/item?id=JTNB_1990_2_2_349_0>

(C) Université Bordeaux 1, 1990, tous droits réservés.

L'accès aux archives de la revue "Séminaire de Théorie des Nombres de Bordeaux » (http://jtnb.cedram.org/) implique l'accord avec les conditions générales d'utilisation (http://www.numdam.org/conditions). Toute utilisation commerciale ou impression systématique est constitutive d'une infraction pénale. Toute copie ou impression de ce fichier doit contenir la présente mention de copyright.

\section{Numdam}

Article numérisé dans le cadre du programme

Numérisation de documents anciens mathématiques

http://www.numdam.org/ 
Séminaire de Théorie des Nombres, Bordeaux 2 (1990), 349-363

\title{
On Taylor's conjecture for Kummer orders.*
}

\author{
by Philippe Cassou-Noguès and Anupam Srivastav
}

\section{Introduction}

Let $\overline{\mathbf{Q}}$ denote the algebraic closure of $\mathbb{Q}$ in $\mathbb{C}$ and let $\bar{O}$ be the ring of algebraic integers of $\bar{Q}$. For a number field $F \subseteq \bar{Q}$ we denote by $O_{F}$ its ring of algebraic integers and we set $\Omega_{F}=\operatorname{Gal}(\overline{\bar{Q}} / F)$.

Let $K$ be a quadratic imaginary number field, $L$ a finite extension of $K$ and $(E / L)$ be an elliptic curve, defined over $L$, with everywhere good reduction and admitting complex multiplication by $O_{K}$.

Let $\mathfrak{A}=(a)$ denote a non-zero integral $O_{K}$-ideal. Let us write $G=G(\mathfrak{A})$ for the subgroup of points in $E(\overline{\mathbb{Q}})$ that are killed by all elements of $\mathfrak{A}$. For $P \in E(L)$, we set

$$
G_{P}=G_{\Gamma}(\mathfrak{A})=\{R \in E(\overline{\mathbb{Q}}):[a] R=P\}
$$

the corresponding $G$-space of points on $E$. We define the corresponding Kummer algebra by

$$
L_{P}=L_{P}(\mathfrak{A})=\operatorname{Map}\left(G_{P}, \overline{\mathbb{Q}}\right)^{\Omega_{L}}
$$

where the addition and multiplication are given value-wise on $\Omega_{I}$. maps from $G_{P}$ to $\overline{\mathbb{Q}}$. In [T] M.-J. Taylor considered the $O_{I \text {-algebra } \mathcal{B} \text { which }}$ represents the $O_{I}$-group scheme of $\mathfrak{A}$ points of $E$. In fact $\mathcal{B}$ is an $O_{T}$. Hopf order in the $L$-algebra $L_{O}=\operatorname{Map}(G, \bar{Q})^{S_{2} \tau}$. where $O$ is the origin of $E$. The $O_{I}$-Cartier dual of $\mathcal{B}$ is an $O_{I \text { - }}$-order in the dual algebra $\mathcal{A}=(\overline{\mathbb{Q}}[G])^{\Omega_{I} \text {. that }}$ we denote by $\Lambda$. Taylor $[\mathrm{T}]$ defined the Kummer order $\tilde{O}_{P}$ as the largest $\Lambda$-module contained in $O_{\Gamma}$ the integral closure of $O_{I}$ in $L_{P}$. He showed that $\tilde{O}_{P}$ is a locally free $\Lambda$-module. We write $\left(\tilde{O}_{P}\right)$ for its class in $C \ell(\Lambda)$, the class group of locally free $\Lambda$-modules.

*This work was done while the second named author was visiting lecturer at Bordeaux University. He wishes to express his gratitude to the University for their hospitality.

Manuscrit reçu le 7 mai 1990 
In [T] the map $\psi: E(L) \rightarrow C \ell(\Lambda)$, given by $\psi(P)=\left(\tilde{O}_{P}\right)$ is shown to be a group homomorphism. Moreover it follows from the definition of $\tilde{O}_{P}$ that $[a] E(L) \subset K e r \psi$. Taylor conjectured in [T] :

(1-3) Conjecture. For any non-zero principal $O_{K^{-} \text {-ideal, }}$

$$
E(L)_{\text {torsion }} \subset K e r \psi .
$$

We remark that in [S-T] the above framework was generalised to include the case of non principal $O_{K}$-ideals.

Let $w_{K}$ denote the number of roots of unity of $K$. The above conjecture was proved in [S-T] under the hypothesis that the ideal $\mathfrak{A}$ be coprime to $w_{K}$. In this article we consider the conjecture for the case where $|G|=2$. We now assume that there is a principal prime ideal $\mathfrak{p}=(\pi)$ dividing 2 . Moreover we assume that $\mathfrak{p}$ is either ramified or split in $(K / \mathbb{Q})$ and that $K \neq \mathbb{Q}(\sqrt{-1})$. We set $\mathfrak{A}=\mathfrak{p}$, so that $G=E[\pi]$ and $|G|=2$. By the theory of complex multiplication we can also deduce that $G \subset E[2] \subset E(L)$.

Therefore $\mathcal{A}=L[G]$ and $\mathfrak{B}=\operatorname{Map}(G, L)$. From [T], Proposition 1, we conclude that the order $\Lambda$, in the present case, is given by

$$
\Lambda=1_{G_{i}} . O_{I}+\left(\pi^{-1} \sigma_{G}\right) O_{I}
$$

where $\sigma_{G}=\sum_{g \in G} g$.

Let $\mathfrak{M}$ denote the unique maximal $O_{I}$-order of $L[G]$. As usual, we denote by $D(\Lambda)$ the kernel of the extension map $e: C \ell(\Lambda) \rightarrow C \ell(\mathfrak{M})$. We define the homomorphism $\psi^{\prime}: E(L) \rightarrow C \ell(\mathfrak{M})$ to be the composite map $e \circ \psi$. For $P \in E(L)$, it is shown in [T] that $|G|$ annihilates $\psi(P)$. Thus, in the present case, $\psi(P)^{2}=1$ in $C \ell(\Lambda)$ and $\psi^{\prime}(P)^{2}=1$ in $C \ell(\mathfrak{M})$. In the second section we shall prove :

Theorem 1. Let $\mathfrak{p}=(\pi)$ be a ramified or split principal prime ideal dividing $2 O_{K}$. Moreover, assume that $E[4] \subset E(L)$. Then for $G=E[\pi]$,

$$
E(L)_{\text {torsion }} \subseteq K \operatorname{Rer}\left(\psi^{\prime}\right) .
$$

Let $\Phi$ denote the quotient map : $O_{I} \rightarrow O_{I} / \bar{\pi} O_{I}$. where $\bar{\pi}$ is the complex conjugate of $\pi$. We denote the image of $O_{I}^{*}$. under $\Phi$ by $\operatorname{Im} O_{I}^{*}$. In section 2 we also calculate $D(\Lambda)$,

Theorem 2. The group kernel is given by

$$
D(\Lambda)=\left(O_{I_{.}} / \bar{\pi} O_{I_{.}}\right)^{*} / \operatorname{Im} O_{I_{L}}^{*} .
$$


The main aim of section 3 is to treat cases where $E[4]$ is not contained in $E(L)$.

We first assume that 2 is split in $(K / Q)$; we denote by $\mathfrak{p}=(\pi)$ a prime ideal of $K$ above 2. We now fix a fractional ideal $\Omega$ of $K$, viewed as a $\mathbb{C}$ lattice, and a 4-division point $\nu$ of $\mathbb{C} / \Omega$ such that $2 \nu$ has annihilator $2 O_{K}$. Corresponding to the pair $(\Omega, \nu)$ we define the "minimal Fueter model" as the elliptic curve $E$ given by :

$$
y^{2}+\sqrt{t} x y=x^{3}+x
$$

where $t=t_{\Omega, \nu}=12 \wp_{\Omega}(2 \nu) /\left(\wp_{\Omega}(\nu)-\wp_{\Omega}(2 \nu)\right)$. We let $L=K(\sqrt{t})$. Our model is then defined over $L$. From $\left[C N-T_{2}\right], I X,(5-4)$, we know that $K(t)=K(4)$, the ray class field $\bmod 4 O_{K}$. Moreover, since 2 is split in $(K / \mathbb{Q})$, we know that $t^{2}-2^{6}$ is a unit, $\left[C N-T_{2}\right], I X,(5-10)$. Therefore $E$ has good reduction everywhere. One can check, using classfield theory, that $E[\pi] \subset E(L)$. We let $Q$ be the primitive $\pi$-division point of $E$. We now assume that $E\left[\pi^{2}\right] \not \subset E(L)$. We consider the map $h: G_{\mathcal{Q}} \rightarrow \bar{O}$ défined by $h(R)=y(R)$, for $R \in G_{Q}$. It will be proved that $h$ lies in $\tilde{O}_{Q}$.

Next we consider the Swan module $\left(\sqrt{t}, \pi^{-1} \sigma_{G}\right) \Lambda$. Since $t^{2}-2^{6}$ is a unit, $\sqrt{t}$ is relatively prime to $|G|=2$. Then this module is a locally free ideal of $\Lambda$ (cf. [U],[S]).

THEOREM 3. Let $Q$ be the primitive $\pi$-division point of the minimal Fueter curve $E$. Then

$$
\sqrt{t} \tilde{O}_{\mathcal{Q}}=h\left(\sqrt{t}, \pi^{-1} \sigma_{G}\right) \Lambda
$$

One can observe that the Swan module is the obstruction to the $\Lambda$-freeness of $\tilde{O}_{\mathcal{Q}}$. As a consequence of Theorem 2 and Theorem 3 we obtain :

Corollary 1. Under the hypothesis of Theorem 3,E $(L)_{\text {torsion }} \subseteq K e r \psi$ if and only if there exists a unit $u$ of $L$ such that $\sqrt{t} \equiv u \bmod \bar{\pi} O_{I}$.

Proof. Since $E\left[\pi^{2}\right] \not \subset E(L)$ the inclusion $E(L)_{\text {torsion }} \subseteq K e r \psi$ is equivalent with $\psi(Q)=1$, (see section 2). By Theorem 3 we know that $\psi(Q)=1$ if and only if $\left(\sqrt{t}, \pi^{-1} \sigma_{G}\right) \Lambda$ is a free $\Lambda$-module. Since we know that the element of $C \ell(\Lambda)$ défined by $\left(\sqrt{t}, \pi^{-1} \sigma_{G}\right) \Lambda$ belongs to $D(\Lambda)$ and is represented by $\sqrt{t}$, the conclusion follows Theorem 2 . 
It will be obviously very interesting to know wether the condition of the corollary is always satisfied. In section 4 we checked that the condition is fulfilled when $K=\mathbb{Q}(\sqrt{-7})$.

Acknowledgement : The authors wish to thank J. Martinet for providing useful computer calculations of certain units for section 4 .

\section{Proof of Theorems 1 and 2.}

We keep the notations of section 1 . Let $m$ be the largest positive integer such that $E\left[\pi^{m}\right] \subset E(L)$. We know that $[\pi] E(L) \subset K e r \psi \subset K e r \psi^{\prime}$. Therefore, in order to prove Theorem 1, it suffices to show that

$$
E\left[\pi^{m}\right]-E\left[\pi^{m-1}\right] \subset K e r \psi^{\prime}
$$

Let us now fix $Q \in E(L)$ such that $G_{Q} \not \subset E(L)$. In this case $L_{Q}$ can be identified with $L(Q)$, the field generated over $L$ by the coordinates of all points of $G_{\mathcal{Q}}$. Of course, now $[L(Q): L]=2$. Let $R \in E(\overline{\mathbb{Q}})$ be such that

$$
\pi R=Q
$$

Then the map :

$$
\begin{gathered}
\operatorname{Gal}(L(Q) / L) \rightarrow G \\
\omega \rightarrow R^{\omega}-R
\end{gathered}
$$

induces a group isomorphism which is independent of the particular choice of $R$. We may identify these two groups. Let $\gamma$ be the non trivial element of $G$.

Proof of Theorem 1.

The proof splits in two steps.

(I) Preliminary step

Let $\hat{G}$ denote the group of characters of $G$. We have an isomorphism

$$
\theta: C \ell(\mathfrak{M}) \simeq \prod_{\chi \in \dot{G}_{i}} C \ell\left(O_{I_{i}}\right)
$$

For $y \in C \ell(\mathfrak{M})$ we write $\theta_{\chi}(y)$ to denote its projection on the $\chi$-component $C \ell\left(O_{T}\right)$. Now $G$ acts as automorphisms on $L(Q)$. We write this action exponentially. For $\chi \in \hat{G}$ and $b \in \operatorname{Map}\left(G_{\mathcal{Q}}, \overline{\mathbb{Q}}\right)$, the Lagrange resolvent of $b$ is defined by

$$
(b \mid \chi)=\sum_{g \in G} b^{g} \chi\left(g^{-1}\right)
$$


Proposition 1. Let $\chi \in \hat{G}$ and $y \in L(Q)$ be such that $y^{g}=y \cdot \chi(g)$, $\forall g \in G$. Then there exists a fractional ideal $I(\chi)$ of $L$ whose class in $C \ell\left(O_{I}\right)$ is independent of the choice of $y$, such that $y^{2} O_{I}=I(\chi)^{2}$. Moreover, $\theta_{\chi}\left(\psi^{\prime}(Q)\right)=[I(\chi)]^{-1}$.

Proof. Clearly the class of $I(\chi)$ does not depend on the choice of $y$. We may, therefore, take $y=\pi^{-1}(d \mid \chi)$ where $d$ generates a normal basis of $L(Q)$ over $L$. From [T], Proposition 6 and Theorem 3, we deduce that there exists a fractional ideal $I(\chi)$ of $L$ such that $\theta_{\chi}\left(\psi^{\prime}(Q)\right)=[I(\chi)]^{-1}$ and $I(\chi) O_{I .(Q)}=\pi^{-1}(d \mid \chi) O_{I .(Q)}$.

Corollary 2. The following statements are equivalent

i) $\psi^{\prime}(Q)=1$

ii) There exists $y \in L(Q) \backslash L$ such that $y^{2} \in L$ and $y^{2} O_{I}$ is a square of a principal $O_{I}$-ideal.

iii) There exists a unit $u \in L$ such that $L(Q)=L(\sqrt{u})$.

\section{(II) Construction of a unit.}

Let us now assume that $E[4] \subset E(L)$ and fix $Q \in E\left[\pi^{m}\right]$. Therefore, in this case $m>1$. We consider a general Weierstrass model of $E$ defined over $L$. Let us fix $R \in G_{Q}$. Let $S$ be the primitive $\pi$-division point and $V$ a primitive 4-division point of $E(L)$. As $G_{Q} \not \subset E(L)$, the points [2]R and [2] $(R+V)$ are both distinct from $S$. Thus $x(R)^{\gamma}=x(R+S) \neq x(R)$ and $x(R+V)^{\gamma}=x(R+V+S) \neq x(R+V)$.

We then have

$$
L(Q)=L(x(R))=L(x(R+V)) .
$$

Thus, by the theorem of Fueter-Hasse, [CN-T 2, IX]

$$
L(Q)=\left\{\begin{array}{lc}
L . K\left(\mathfrak{p}^{m+1}\right) & \text { if } 2 \text { is ramified in }(K / \mathbb{Q}) \\
L . K\left(4 \mathfrak{p}^{m-1}\right) & \text { if } 2 \text { is split in }(K / \mathbb{Q})
\end{array}\right.
$$

where $K(f)$ denotes the $K$-ray class field mod $f$ for any $O_{K^{-}}$ideal $f$.

Next we fix an analytic parametrisation

$$
\mathbb{C} / \Omega \stackrel{\sim}{\rightarrow} E(\mathbb{C})
$$

for a certain lattice $\Omega$ of $\mathbb{C}$. 
We now set :

$$
A_{\mathcal{Q}}=\left\{\begin{array}{l}
\frac{h_{\Omega}(R)-h_{\Omega}(R+S)}{h_{\Omega}(Q)-h_{\Omega}(Q+S)}, \quad \text { if } 2 \text { is ramified in }(K / \mathbb{Q}) \\
\frac{h_{\Omega}(R+V)-h_{\Omega}(R+V+S)}{h_{\Omega}(Q+V)-h_{\Omega}(Q+V+S)}, \quad \text { if } 2 \text { is split in }(K / \mathbb{Q})
\end{array}\right.
$$

where $h_{\Omega}$ is the first Weber's function. Once again from the theory of complex multiplication we know that $A_{Q} \in K\left(\mathfrak{p}^{m+1}\right)\left(\operatorname{resp} . K\left(4 \mathfrak{p}^{m-1}\right)\right)$ if 2 is ramified (resp. split) in $(K / Q)$. Moreover we obtain that

$$
\left\{\begin{aligned}
K\left(\mathfrak{p}^{m+1}\right) & =K\left(\mathfrak{p}^{m}\right)\left(A_{\mathcal{Q}}\right), \text { if } 2 \text { is ramified in }(K / \mathbb{Q}) \\
K\left(4 \mathfrak{p}^{m-1}\right) & =K\left(4 \mathfrak{p}^{m-2}\right)\left(A_{\mathcal{Q}}\right), \text { if } 2 \text { is split in }(K / \mathbb{Q})
\end{aligned}\right.
$$

From (2.3) we then deduce that

$$
L(Q)=L\left(A_{\mathcal{Q}}\right) \text { and } A_{\mathcal{Q}}^{2} \in L
$$

Let $\wp_{\Omega}$ be the Weierstrass $\wp$ function for $\Omega$. From the definition of $h_{\Omega}$ we deduce that

$$
A_{Q}=\left\{\begin{array}{l}
\frac{\xi_{\Omega}(R)-\xi_{\Omega}(R+S)}{\xi_{\Omega}(Q)-\xi_{\Omega}(Q+S)}, \text { if } 2 \text { is ramified in }(K / Q) \\
\frac{\xi_{\Omega}(R+V)-\xi_{\Omega}(R+V+S)}{\xi_{\Omega}(Q+V)-\xi_{\Omega}(Q+V+S)}, \text { if } 2 \text { is split in }(K / Q)
\end{array}\right.
$$

Let $\mathcal{H}$ denote the upper half plane. Let $\tau \in \mathcal{H}$ be such that $\Omega=\lambda(\mathbb{Z} \tau+\mathbb{Z})$ for some $\lambda \in \mathbb{C}^{*}$. For $z \in \mathcal{H}$ we write $\Omega_{z}=\mathbb{Z} z+\mathbb{Z}$. For $a \in(\mathbb{Q} / \mathbb{Z})^{2}$ we choose the unique representative $\left(a_{1}, a_{2}\right) \in \mathbb{Q}^{2}$ with $a_{1}, a_{2} \in[0,1[$. We write $a z=a_{1} z+a_{2}$. We define $r$ (resp.s, resp.v,resp.q) in $(\mathbb{Q} / \mathbb{Z})^{2}$ such that $\lambda(r \tau)($ resp. $\lambda(s \tau)$, resp. $\lambda(v \tau)$, resp. $\lambda(q \tau))$ represents $R($ resp.S, resp. $V$,resp. $Q)$ in $\mathbb{C}$ mod. $\Omega$. We now consider functions $F(r, q, s)$ and $G(r, q, s, v)$ defined by

$$
F(r, q, s)(z)=\frac{\wp_{\Omega_{z}}(r z)-\wp_{\Omega_{z}}(r z+s z)}{\wp_{\Omega_{z}}(q z)-\wp_{\Omega_{z}}(q z+s z)}
$$

and

$$
\begin{gathered}
G(r, q, s, v)(z)=\frac{\wp_{\Omega_{z}}(r z+v z)-\wp_{\Omega_{z}}(r z+v z+s z)}{\wp_{\Omega_{z}}(q z+v z)-\wp_{\Omega_{z}}(q z+v z+s z)} \\
A_{Q}= \begin{cases}F(r, q, s)(\tau) & \text { if } 2 \text { ramified } \\
G(r, q, s, v)(\tau) & \text { if } 2 \text { splits. }\end{cases}
\end{gathered}
$$


Functions $F$ and $G$ are modular Weierstrass units of a level which is an appropriate power of 2 .

When $f$ and $g$ are functions defined on $\mathcal{H}$ we write

$$
f \approx g
$$

if there exist integers $n$ and $m$ such that $f^{n} / g^{m}$ is a modular function, which is a unit over $\mathbb{Z}$.

For $a \in(\mathbb{Q} / \mathbf{Z})^{2}$ we introduced in $\left[C N-T_{1}\right],(2-7)$, a function $\tilde{\Psi}(a)$ defined on $\mathcal{H}$. In fact an appropriate power of $\tilde{\Psi}(a)$ is a ratio of Deuring modular units. From [CN-T $]$, Proposition 2-8, we obtain

Lemma 1. There are equivalences

$$
F(r, q, s) \approx \frac{\tilde{\Psi}^{2}(q) \tilde{\Psi}^{2}(q+s) \tilde{\Psi}(2 r+s)}{\tilde{\Psi}^{2}(r) \tilde{\Psi}^{2}(r+s) \tilde{\Psi}(2 q+s)}
$$

and

$$
G(r, q, s, v) \approx \frac{\tilde{\Psi}^{2}(q+v) \tilde{\Psi}^{2}(q+s+v) \tilde{\Psi}(2 r+2 v+s)}{\tilde{\Psi}^{2}(r+v) \tilde{\Psi}^{2}(r+v+s) \tilde{\Psi}(2 q+2 v+s)}
$$

We now show :

LEMMA 2. (i) If 2 is ramified in $(K / Q)$, then $F(r, q, s)(\tau)$ is a unit.

(ii) If 2 is split in $(K / Q)$ and $m>2$, then $G(r, q, s, v)(\tau)$ is a unit.

Proof (i) Let 2 be ramified in $(K / Q)$ and suppose $m=2 t, t>1$ (if $m$ is odd the proof is similar). Then, $q \tau($ resp. $(q+s) \tau$, resp. $(2 q+s) \tau$, resp.r $\tau$, resp. $(r+s) \tau$, resp. $(2 r+s) \tau)$ defines a primitive $\mathfrak{p}^{2 t}\left(\right.$ resp.p $\mathfrak{p}^{2 t}$, resp. $\mathfrak{p}^{2(t-1)}$, resp. $\mathfrak{p}^{2 t+1}$, resp. $\mathfrak{p}^{2 t+1}$, resp. $\left.\mathfrak{p}^{2 t-1}\right)$-division point of $\mathbb{C} / \Omega_{\tau}$.

For two algebraic numbers $a, b$ we write $a \sim b$ if $a b^{-1}$ is a unit. From $\left[C N-T_{1}\right]$, Proposition 3-5, we deduce that

$$
\left\{\begin{array}{l}
\tilde{\Psi}(q)(\tau) \sim \tilde{\Psi}(q+s)(\tau) \sim 2^{\left(2^{-2 t}\right)} \\
\tilde{\Psi}(r)(\tau) \sim \tilde{\Psi}(r+s)(\tau) \sim 2^{\left(2^{-2 t-1}\right)} \\
\tilde{\Psi}(2 q+s)(\tau) \sim 2^{\left(2^{2-2 t}\right)} \\
\tilde{\Psi}(2 r+s)(\tau) \sim 2^{\left(2^{1-2 t}\right)}
\end{array}\right.
$$


Thus from Lemma 1 and (2-10) we conclude that $F(q, r, s)(\tau)$ is a unit.

(ii) Now suppose that 2 is split in $(K / \mathbb{Q})$ and $m>2$. Then $(q+v) \tau$ and $(q+v+s) \tau$ are primitive $\mathfrak{p}^{m} \overline{\mathfrak{p}}^{2}$ division points ; $(r+v) \tau$ and $(r+$ $v+s) \tau$ are primitive $\mathfrak{p}^{m+1} \overline{\mathfrak{p}}^{2}$-division points. Moreover $(2 r+2 v+s) \tau$ (resp. $(2 q+2 v+s) \tau)$ is a primitive $\mathfrak{p}^{m} \overline{\mathfrak{p}}\left(\right.$ resp.p $\left.\mathfrak{p}^{m-1} \overline{\mathfrak{p}}\right)$-division point. Since these points are primitive of composite order, it follows from $\left[C N-T_{1}\right]$, Proposition 3-5, that each factor in the right hand side of the equivalence in Lemma 1 gives a unit when evaluated at $\tau$. From (2-9) and Lemma 2 we now conclude that $A_{\mathcal{Q}}$ is a unit. Therefore Theorem 1 is proved, via Corollary 2, except in the case where 2 is split in $(K / \mathbb{Q})$ and $m=2$. We can, nevertheless, treat this case in a similar fashion by replacing $A_{\mathcal{Q}}$ by $A_{Q}^{1}$ given by

$$
A_{Q}^{1}=\pi^{-1}\left(P_{\Omega 2}(R+V)-P_{\Omega 2}(R+V+S)\right)
$$

where $P_{\Omega}$ is the function considered by Schertz [Sh]. We know that

$$
A_{\mathcal{Q}}^{1}=\kappa\left(h_{\Omega 2}(R+V)-h_{\Omega 2}(R+V+S)\right)
$$

where $\kappa \in K(1)$. We thus have $A_{\mathcal{Q}}^{1} \in L_{\mathcal{Q}} \backslash L$ and $\left(A_{\mathcal{Q}}^{1}\right)^{2} \in L$. We now deduce from [Sch], (12) and Satz 3 , that $A_{\mathcal{Q}}^{1}$ is a unit. This now completes the proof of Theorem 1 .

Proof of Theorem 2. We recall that the order $\Lambda$ is explicitly given by (1-4). Let us consider the fiber product of orders

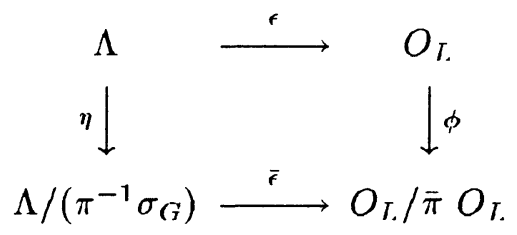

where $\eta$ and $\phi$ are the quotient maps, $\epsilon$ is the augmentation map and $\bar{\epsilon}$ is induced by $\epsilon$. Using the Mayer-Vietoris sequence of Reiner-Ullom, [S], [U], we obtain an exact sequence of groups and homomorphisms.

$$
O_{I .}^{*} \times\left(\Lambda /\left(\pi^{-1} \sigma_{G}\right)\right)^{*} \stackrel{\phi \bar{\epsilon}^{-1}}{\longrightarrow}\left(O_{I .} / \bar{\pi} O_{L}\right)^{*} \stackrel{\delta}{\longrightarrow} D(\Lambda) \rightarrow\{1\}
$$

where $\delta$ is the connecting homomorphism. 
We also need to observe that

$$
D\left(O_{I}\right)=D\left(\Lambda /\left(\pi^{-1} \sigma_{G}\right)\right)=\{1\}
$$

Moreover, for $s$ coprime with $\bar{\pi}, \delta\left(s \bmod \bar{\pi} O_{I}\right)$ is given by the class of the corresponding Swan module $\left(s, \pi^{-1} \sigma_{G}\right) \Lambda$. Since $O_{I}$. and $\Lambda /\left(\pi^{-1} \sigma_{G}\right)$ can be naturally identified as rings, we conclude that

$$
D(\Lambda)=\left(O_{I .} / \bar{\pi} O_{I .}\right)^{*} / \operatorname{Im} O_{I .}^{*}
$$

\section{Minimal Fueter model}

We recall in this section that $\mathfrak{p}=(\pi)$ is a principal, prime ideal of $K$, above 2 , which is split in $(K / \mathbb{Q})$. Moreover we suppose that $E[\pi] \subset E(L)$ and $E\left[\pi^{2}\right] \not \subset E(L)$. We let $\Omega$ be a fractional ideal of $K$ and $\nu$ a primitive $4 O_{K}$-division point of $C / \Omega$.

In $\left[C N-T_{2}\right]$ a Fueter elliptic curve was considered, corresponding to the pair $(\Omega, \nu)$, given by

$$
y^{2}=4 x^{3}+t x^{2}+4 x
$$

with

$$
t=12 \wp_{\Omega 2}\left((2 \nu) /\left(\wp_{\Omega 2}(\nu)-\wp_{\Omega}(2 \nu)\right) .\right.
$$

In fact one defines a complex analytic isomorphism between $C / \Omega$ and the complex points of this curve by considering

$$
z \rightarrow \begin{cases}\left(T(z), T_{1}(z), 1\right) & \text { if } z \neq 2 \nu \\ (0,1,0) & \text { if } z=2 \nu\end{cases}
$$

where $T$ and $T_{1}$ are Fueter's elliptic functions, [CN- $\left.T_{2}\right], I V$. The minimal Fueter model $E$ is obtained from (3.1) by the change of coordinates

$$
(x, y) \rightarrow(x, \sqrt{t} x+2 y)
$$

From (3-2) and (3-3) we deduce an isomorphism between $\mathbb{C} / \Omega$ and the C-points of $E$ given by

$$
z \rightarrow \begin{cases}(T(z), U(z), 1) & \text { if } z \neq 2 \nu \\ (0,1,0) & \text { if } z=2 \nu\end{cases}
$$

where $U(z)=(1 / 2)\left(T_{1}(z)-\sqrt{t} T(z)\right)$. 
We remark that $0=(0,0,1)$ is taken to be the identity of the group law. It is also worth remarking that $i \in K(t)$. We set $A=(i, 0,1)$. It is worth to notice that, using the theory of complex multiplication, one can show that $A \in E(L)$ and has infinite order. Let $\alpha$ be the parameter of $A$ in $\mathbb{C} / \Omega$ under the isomorphism (3-4).

The divisor of $T$ is given by

$$
(T)=2(0)-2(2 \nu)
$$

From $\left[C N-T_{2}\right]$,IV we know that

$$
\begin{gathered}
T(z) \cdot T(z+2 \nu)=1 . \\
T_{1}(z+2 \nu)=-T_{1}(z) / T^{2}(z) .
\end{gathered}
$$

Therefore, since $T$ is an even function and $T_{1}$ is an odd function, we deduce that

$$
U(2 \nu-z)=U(z) / T^{2}(z) .
$$

Moreover, the elliptic function $U$ has divisor

$$
(U)=(0)+(\alpha)+(2 \nu-\alpha)-3(2 \nu) .
$$

We denote by $N$ the point of $E(\bar{Q})_{\text {torsion }}$ defined by $\nu$. Let $Q$ be the primitive $\pi$-division point of $E$. We fix a point $R \in G_{Q}$ and denote by $\rho$ its parameter in $C / \Omega$.

Now $R+Q=-R$, therefore $G_{Q}=\{R,-R\}$.

Thus, $x(R)^{\gamma}=x(R+Q)=x(-R)=x(R)$. Then $L(Q)=L(y(R))=$ $L\left(T_{1}(\rho)\right)=L(D(\rho))$ where $D(\rho)=T_{1}(\rho) / T(\rho)$.

From $\left[C N-T_{2}\right]$, IX, (6-7) we know that $D^{4}(\rho)=t^{2}-2^{6}$, which is a unit. Since $D^{2}(\rho) \in L$ we conclude from Corollary 2 that $\psi^{\prime}(Q)=1$.

Until the end of this section the $x$ and $y$ coordinates are those of model (1-5).

We now want to study $\psi(Q)$. First, we have

Lemma 3. Let $\mathfrak{P}$ be a prime ideal of $O_{K^{*}}$. Let $P \in E(\bar{Q})_{\text {torsion }}$ be such that $\left.\{P,[2] N-P\} \bigcap_{n>0} E\left[\mathfrak{P}^{n}\right]\right)=\phi$. Then $x(P)$ is a $\mathfrak{P}$-unit (i.e. unit at all primes dividing $\mathfrak{P})$.

Proof. We first observe that for any $P \in E(\bar{Q}), P \neq[2] N, x(P)$ is a $\mathfrak{P}$ integer if and only if $y(P)$ is a $\mathfrak{P}$-integer. Under the given hypothesis both 
$x(P)$ and $y(P)$ are well defined and are non zero. Since $x(P) \cdot x([2] N-P)=$ 1 , it suffices to show that $x(P)$ is a $\mathfrak{P}$-integer.

Let $M$ be a finite extension of $L$ such that $\{P,[2] N-P\} \subset E(M)$. Suppose $x(P)$ is not a $\mathfrak{P}$-integer. Then there exists $\mathfrak{P}_{M}$, a maximal $O_{M}$-ideal, with $\mathfrak{P}_{M} \cap O_{K}=\mathfrak{P}$ and $v(x(P))<0$ where $v$ denote the standard valuation on the completion of $M$ at $\mathfrak{P}_{M}$. From the equation of the minimal Fueter model $E$ we see that $2 v(y(P))=3 v(x(P))$. Thus, under the reduction mod $\mathfrak{P}_{M}, P$ is mapped onto $(0,1,0)$. This means that [2]N-P is in the kernel of reduction modP $\mathfrak{P}_{M}$ which is impossible since the set of torsion points in the kernel of reduction is precisely $\bigcup_{n>0} E\left[\mathfrak{P}^{n}\right]$.

LEMMA $4 . x(R) \sim \pi$

Proof. Since $R$ is a primitive $\pi^{2}$-division point of $E,[2] N-R$ is a torsion point of composite order. From Lemma 3 we conclude that $x(R)$ is a unit outside the prime divisors of $\mathfrak{p}=(\pi)$. For a prime $\mathfrak{P}$ of $L(Q)$ that divides $\mathfrak{p}$, using that $R$ is a primitive $\pi^{2}$-division point in the kernel of reduction mod $\mathfrak{P}_{I .(\mathcal{Q})}$ and that $x(R) / y(R)$ is the parameter of $R$ in the associated formal group we can find the valuation $v_{\mathfrak{P}_{I}(\mathcal{Q})}(x(R))$.

Remark : Lemma 3 and 4 can both be proved using the technique of modular functions as developed in section 2, Lemma 1 and 2.

It follows from the equation of $E$ that $y(R)^{2} / \pi$ is an algebraic integer and a $\mathfrak{p}$-unit.

We now consider the map

$$
\begin{aligned}
h: G_{\mathcal{Q}} & \rightarrow \overline{\mathbb{Q}} \\
& M \rightarrow y(M) .
\end{aligned}
$$

Proposition 2.

i) The map $h$ lies in $\tilde{O}_{\mathcal{Q}}$

ii) Let $\chi \in \hat{G}$ and $M \in G_{\mathcal{Q}}$, then

$$
(h \mid \chi)(M) \sim \begin{cases}\sqrt{t} x(M), & \text { if } \chi \text { is trivial } \\ x(M) & \text { otherwise. }\end{cases}
$$

Proof. We first prove (ii). Since $x$ is an even function and $T_{1}$ an odd function, we obtain from the definition of $h$ and (3-4) 


$$
(h \mid \chi)(M)=\left\{\begin{array}{l}
-\sqrt{t} x(m), \text { if } \chi \text { is the identity character } \\
T_{1}(m) \text { otherwise }
\end{array}\right.
$$

where $m$ is the parameter of $M$ in $\mathbb{C} / \Omega$. Since $m= \pm \rho$ we have $T_{1}(m)=$ $\pm D(\rho) x(M)$ and then, since $D(\rho)$ is a unit, $T_{1}(m) \sim x(M)$. We now prove i). By lemma 4 it is evident that $h \in O_{Q}$. Since

$$
\Lambda=1_{G} O_{I .}+\left(\pi^{-1} \sigma_{G}\right) O_{I},
$$

we need only check that $h .\left(\pi^{-1} \sigma_{G}\right) \in O_{Q}$. For $M \in G_{Q}$ we obtain

$$
h\left(\pi^{-1} \sigma_{G}\right)(M)=\pi^{-1}(h \mid \epsilon)(M)=-\pi^{-1} \sqrt{t} \cdot x(M)
$$

where $\epsilon$ is the identity character. Using Lemma 4 we conclude that $h\left(\pi^{-1} \sigma_{G}\right)(M) \in \bar{O}$. Hence $h$ lies in $\tilde{O}_{\mathcal{Q}}$.

Proof of Theorem 3. The proof is similar to that of Theorem 5 in [S-T]. We must show the equality locally. For each prime $\mathfrak{P}$ of $L$ we write

$$
\begin{aligned}
\tilde{O}_{\mathcal{Q}, \mathfrak{P}} & =\theta_{\mathfrak{P}} \Lambda_{\mathfrak{P}} \\
\left(\sqrt{t}, \pi^{-1} \sigma_{G}\right) \Lambda_{\mathfrak{P}} & =a_{\mathfrak{P}} \Lambda_{\mathfrak{P}}
\end{aligned}
$$

where $\theta_{\mathfrak{P}}\left(\right.$ resp. $\left.a_{\mathfrak{P}}\right)$ belongs to $\tilde{O}_{\mathcal{Q}, \mathfrak{P}}\left(\right.$ resp. $\left.\Lambda_{\mathfrak{P}}\right)$. From Theorem 3 of $[\mathrm{T}]$ we know that for $M \in G_{Q}$ and $\chi \in \hat{G}$ we have

$$
\left(\theta_{\mathfrak{P}} \mid \chi\right)(M) \sim \pi .
$$

We let $\chi$ act on $L_{\mathfrak{P}}[G]$ by $L_{\mathfrak{P}}$-linearity. We first observe that $\chi\left(\Lambda_{\mathfrak{P}}\right)=O_{I . \mathfrak{p}}$. Then, by looking at $\chi\left(a_{\mathfrak{P}} \Lambda_{\mathfrak{P}}\right)$, we obtain

$$
\chi\left(a_{\mathfrak{P}}\right) \sim\left\{\begin{array}{l}
1, \text { if } \chi \text { is the identity character } \\
\sqrt{t} \text { otherwise. }
\end{array}\right.
$$

We now can write

$$
\theta_{\mathfrak{P}} \cdot\left(\sqrt{t} b_{\mathfrak{P}}\right)=h a_{\mathfrak{P}}
$$

with $b_{\mathfrak{P}} \in L_{\mathfrak{P}}[G]$. In order to prove the theorem we must show that $b_{\mathfrak{P}} \in$ $\Lambda_{\mathfrak{P}}^{*}$. Since $h \in \tilde{O}_{\mathcal{Q}, \mathfrak{P}}, h \sqrt{t}$ and $h\left(\pi^{-1} \sigma_{G}\right)$ lie in $\tilde{O}_{\mathcal{Q}, \mathfrak{P}}$. We conclude from (3-10) that $h a_{\mathfrak{P}} \in \tilde{O}_{\mathcal{Q}, \mathfrak{P}}$ and, from (3-13), that $\sqrt{t} b_{\mathfrak{P}} \in \Lambda_{\mathfrak{P}}$. 
For $\chi \in \hat{G}$ we consider the Lagrange resolvent of both sides of (3-13). We obtain

$$
\sqrt{t}\left(\theta_{\mathfrak{P}} \mid \chi\right) \chi\left(b_{\mathfrak{P}}\right)=(h \mid \chi) \chi\left(a_{\mathfrak{P}}\right)
$$

Using Lemma 4, Proposition 2, (3-11) and (3-12), we deduce from (3-14) that $\chi\left(b_{\mathfrak{P}}\right) \sim 1$.

We now consider two cases.

Case 1. $\mathfrak{P}+\sqrt{t}$. In this case $\sqrt{t} b_{\mathfrak{P}} \in \Lambda_{\mathfrak{P}}$ implies that $b_{\mathfrak{P}} \in \Lambda_{\mathfrak{P}}$; so $b_{\mathfrak{P}} \in \Lambda_{\mathfrak{P}}^{*}$, since $\chi\left(b_{\mathfrak{P}}\right)$ is a unit for all $\chi \in \hat{G}$.

Case 2. $\mathfrak{P} \mid \sqrt{t}$. Since $\sqrt{t}$ is coprime with $2, \mathfrak{P}+2$. Then $\Lambda_{\mathfrak{P}}$ is the unique maximal order and $b_{\mathfrak{P}} \in \Lambda_{\mathfrak{P}}^{*}$ since $\chi\left(b_{\mathfrak{P}}\right)$ is a unit for all $\chi \in \hat{G}$.

Remark : If 2 splits in $(K / \mathbb{Q}),(2)=(\pi)(\bar{\pi})$ and $E$ denotes the Fueter minimal model

$$
y^{2}+\sqrt{t} x y=x^{3}+x
$$

then for any number field $L \supset K(\sqrt{t})$ and $G=E[\pi]$ we have that $E(L)_{\text {torsion }} \subset K e r \psi^{\prime}$.

One can easily check that if $E\left[\pi^{2}\right] \subset E(L)$ then $E[4] \subset E(L)$ and we can use the results of section 2 .

\section{Examples}

In this section we consider the set up of section 3 for the particular case of $K=\mathbb{Q}(\sqrt{-7})$.

We set $\pi=(1+\sqrt{-7}) / 2$ and $2=\pi \bar{\pi}$, where $\bar{\pi}$ is the complex conjugate of $\pi$. We note that the class number of $K$ is $1, K(2)=K$ and $[K(4): K]=2$. Since $i \in K(t)=K(4)$ we must have $K(t)=K(i)$. Moreover, since $t^{2}-2^{6}$ is a unit in $K(2)$, we know that $t^{2}-2^{6}= \pm 1$. The possibility $t^{2}-2^{6}=1$ contradicts the fact that $K(t)=K(i)$. Hence $t^{2}=63$ and $L=K(\sqrt[4]{63})$; therefore $L$ is the splitting field of $X^{4}-63$.

We first determine the group kernel $D(\Lambda)$ considered in Theorem 2 .

Proposition 3. $D(\Lambda)=\{1\}$.

Proof. By Theorem 2 we know that

$$
D(\Lambda)=\left(O_{I .} / \bar{\pi} O_{I .}\right)^{*} / \operatorname{Im} O_{I}^{*}
$$


It is easily checked that the ramification index of (2) in $L$ is 4 . Hence the group $\left(O_{I_{L}} / \bar{\pi} O_{I_{i}}\right)^{*}$ is of order 8 . We have to show that $\operatorname{Im}\left(O_{I_{L}}\right)^{*}$ also has order 8 . Let $\alpha=\sqrt[4]{63}$ and $\beta=(1+i) \alpha$. We set $u=(1-i)(1+\pi)+\alpha, v=$ $1-3 \alpha+\alpha^{3} / 3$ and $w=5-2 \beta-12 \pi-2 \pi \beta$. We verify that

$$
\begin{array}{r}
u^{2}=i w, w \cdot(5+2 \beta-12 \pi+2 \pi \beta)=1 \\
v\left(127+45 \alpha+12 \alpha^{2}+17 \alpha^{3} / 3\right)=1
\end{array}
$$

Therefore $u, v$ and $w$ are all units of $L$. We also have

$$
\begin{aligned}
u^{2} & \equiv i \bmod \bar{\pi} O_{I .}, v^{2} \equiv 1 \bmod \bar{\pi} O_{I} \\
i^{2} & \equiv 1 \bmod \bar{\pi} O_{I .}
\end{aligned}
$$

and

$$
\begin{aligned}
& i \not \equiv 1 \bmod \bar{\pi} O_{I .}, \quad v \not \equiv 1 \bmod \bar{\pi} 0_{I}, \\
& v \not \equiv i \bmod \bar{\pi} O_{I}
\end{aligned}
$$

Let $\Phi$ be the quotient map

$$
\Phi: O_{I .} \rightarrow\left(O_{I .} / \bar{\pi} O_{I .}\right)
$$

It follows from (4-2) and (4-3) that $\Phi(u)$ is of order 4 and that $\Phi(v)$ doesn't lie in the subgroup generated by $\Phi(u)$. Hence we must have that the order of $\operatorname{Im}\left(O_{I}^{*}\right)$ is 8 .

We know from section 3 that

$$
L\left(E\left[\pi^{2}\right]\right)=L\left(\left(t^{2}-2^{6}\right)^{1 / 4}\right)=L(\sqrt{i}) .
$$

Therefore :

$$
E(L)_{\text {torsion }} \subset K e r \psi^{\prime}
$$

Hence, from Proposition 3, we conclude

Corollary 3 .

$$
E(L)_{\text {torsion }} \subset K e r \psi
$$

\section{REFERENCES}

[CN-T 1] Ph. CASSOU-NOGUES, M.-J. TAYLOR, Unités modulaires et monogénéité d'anneaux d'entiers, Séminaire de Théorie des Nombres, Paris (1986-1987), 35-63. 
[CN-T 2] Ph. CASSOU-NOGUES, M.-J. TAYLOR, Rings of integers and elliptic functions, Progress in Mathematics 66. Birkhauser, Boston, (1987).

[S] A. SRIVASTAV, A note on Swan modules, Indian Jour. Pure and Applied Math. 20/11 (1989), 1067-1076.

[Sch] SCHERTZ, Konstruktion von Ganzheitsbasen in Strahlklassenkörper über imaginär quadratischen Zahlkörpern, J. de Crelle. à paraître.

[S-T] A. SRIVASTAV, M.-J. TAYLOR, Elliptic curves with complex multiplication and Galois module structure. Invent. Math. 99 (1990), 165-184.

[T] M.-J. TAYLOR, Mordel-Weil groups and the Galois module structure of rings of integers, Mlinois Jour. Math. 32 (3) (1988), 428-452.

[U] S.-V. ULLOM, Nontrivial lower bounds for class groups of integral group rings, Mlinois Jour Math. 20 (1976), 361-371.

Centre de Recherche en Mathématiques de Bordeaux

Université Bordeaux I

C.N.R.S. U.A. 226

U.F.R. de Mathématiques

351 , cours de la Libération

33405 Talence Cedex, FRANCE

and

SPIC Science Fondation

East Coast Chambers

92 GN Chetty road

600017 Madras INDIA. 